PROCEEDINGS OF THE

AMERICAN MATHEMATICAL SOCIETY

Volume 104, Number 1, September 1988

\title{
OPTIMAL PARTITIONING OF A MEASURABLE SPACE
}

\author{
JERZY LEGUT AND MACIEJ WILCZYŃSKI
}

(Communicated by R. Daniel Mauldin)

\begin{abstract}
An $\alpha$-optimal partition of a measurable space according to $n$ nonatomic probability measures is defined. A minmax theorem is used to find a method of obtaining the $\alpha$-optimal partition. An application to a problem of fair division is given.
\end{abstract}

1. Introduction. Let $(\mathscr{X}, \mathscr{B})$ be a measurable space and let $\mu_{1}, \mu_{2}, \ldots, \mu_{n}$ denote nonatomic probability measures defined on the same $\sigma$-algebra $\mathscr{B}$. By an ordered partition $P=\left\{A_{i}\right\}_{i=1}^{n}$ of the measurable space $(\mathscr{X}, \mathscr{B})$ is meant a collection of disjoint subsets $A_{1}, A_{2}, \ldots, A_{n}$ of $\mathscr{X}$ satisfying $A_{i} \in \mathscr{B}$ for all $i \in I:=$ $\{1,2, \ldots, n\}$ and $\bigcup_{i=1}^{n} A_{i}=\mathscr{X}$. Let $\mathscr{P}$ denote the set of all measurable partitions $P=\left\{A_{i}\right\}_{i=1}^{n}$ of $\mathscr{X}$. Suppose that $\alpha \in S:=\left\{s=\left(s_{1}, s_{2}, \ldots, s_{n}\right) \in R: s_{i}>0\right.$ for all $i \in I$ and $\left.\sum_{i=1}^{n} s_{i}=1\right\}$. if

DEFINITION. A partition $P^{\alpha}=\left\{A_{i}^{\alpha}\right\}_{i=1}^{n} \in \mathscr{P}$ is considered to be an $\alpha$-optimal

$$
\min _{i \in I}\left[\alpha_{i}^{-1} \mu_{i}\left(A_{i}^{\alpha}\right)\right]=\sup \left\{\min _{i \in I}\left[\alpha_{i}^{-1} \mu_{i}\left(A_{i}\right)\right]: P=\left\{A_{i}\right\}_{i=1}^{n} \in \mathscr{P}\right\}
$$

where $\alpha_{i}$ is the $i$ th coordinate of $\alpha \in S$.

It is easy to see that the $\alpha$-optimal partition is also Pareto optimal (cf. [8]).

The problem of $\alpha$-optimal partitioning of a measurable space $(\mathscr{X}, \mathscr{B})$ can be interpreted as the well-known problem of fair division of an object $\mathscr{X}$ (e.g. a cake) among $n$ participants (cf. $[\mathbf{2}, \mathbf{5}]$ ). Here, each $\mu_{i}, i \in I$, represents the individual evaluation of sets from $\mathscr{B}$. We also assume in this problem that $\mu_{1}, \mu_{2}, \ldots, \mu_{n}$ are nonatomic probability measures. Dividing the object $\mathscr{X}$ fairly we are interested in giving the $i$ th person a set $A_{i} \subset \mathscr{X}$ such that $\mu_{i}\left(A_{i}\right) \geq 1 / n$ for all $i \in I$.

A simple and well-known method for realizing a fair division (of a cake) for two players is "for one to cut, the other to choose". Steinhaus in 1944 (cf. [5]) asked whether the fair procedure could be found for dividing a cake among $n$ participants for $n>2$. He found a solution for $n=3$ and Banach and Knaster (cf. [5]) showed that the solution for $n=2$ could be extended to arbitrary $n$. Moreover, Knaster [5] proved that if we assume that $\mu_{k} \neq \mu_{j}$ for some $k \neq j$, then there exists a partition $P=\left\{A_{i}\right\}_{i=1}^{n}$ such that $\mu_{i}\left(A_{i}\right)>1 / n$ for all $i \in I$. Urbanik [8] showed the existence of a partition which maximizes $\min _{i \in I}\left[\mu_{i}\left(E_{i}\right)\right]$ over all partitions $P=\left\{E_{i}\right\}_{i=1}^{n} \in \mathscr{P}$. It is easy to verify that this partition is the $\alpha$-optimal if $\alpha_{i}=1 / n$ for each $i \in I$.

Dubins and Spanier [2] obtained for arbitrary $\alpha$ the existence of a partition $P=\left\{A_{i}\right\}_{i=1}^{n}$ such that $\mu_{i}\left(A_{i}\right) \geq \alpha_{i}\left(\mu_{i}\left(A_{i}\right)>\alpha_{i}\right.$ if $\mu_{k} \neq \mu_{j}$ for some $\left.k \neq j\right)$ for each $i \in I$. The $\alpha$-optimal partition $P^{\alpha}$ defined above not only satisfies the

Received by the editors August 20, 1986 and, in revised form, September 23, 1987.

1980 Mathematics Subject Classification (1985 Revision). Primary 90D35, 28B05; Secondary $62 \mathrm{C} 20$. 
condition $\mu_{i}\left(A_{i}\right) \geq \alpha_{i}$ for $i \in I$, but also is proportionally optimal according to the shares $\alpha_{1}, \alpha_{2}, \ldots, \alpha_{n}$ (cf. [8]). Dubins and Spanier showed additionally that there exists a partition which maximizes $\sum_{i=1}^{n} \mu_{i}\left(A_{i}\right)$ and that there is a partition which is optimal in a lexicographic sense. Elton et al. [4] gave an estimation of the number

$$
v^{*}=\sup \left\{\min _{i \in I}\left[\mu_{i}\left(A_{i}\right)\right]: P=\left\{A_{i}\right\}_{i=1}^{n} \in \mathscr{P}\right\}
$$

by suitable inequalities. Their result was improved and generalized for $\alpha$-optimal partitions by Legut [6] and Wilczyński [9].

Our main purpose in this paper is to show how the $\alpha$-optimal partition can be obtained from the theorem of Dvoretzky et al. [3].

2. Main Theorem. Under the assumptions given above, Dvoretzky et al. [3] (cf. [2]) proved the following

THEOREM 1. Let $\bar{\mu}: \mathscr{P} \rightarrow R^{n}$ denote the division vector valued function defined by

$$
\bar{\mu}(P)=\left(\mu_{1}\left(A_{1}\right), \mu_{2}\left(A_{2}\right), \ldots, \mu_{n}\left(A_{n}\right)\right) \in R^{n}, \quad P=\left\{A_{i}\right\}_{i=1}^{n} \in \mathscr{P} .
$$

Then the range $\bar{\mu}(\mathscr{P})$ of $\bar{\mu}$ is convex and compact in $R^{n}$.

To prove our main theorem we make use of the well-known result of Sion (see [1]) which we mention here in the less general form.

THEOREM 2. Let $A$ and $B$ be convex compact sets. Assume that $K(a, b)$ is a continuous function on the Cartesian product $A \times B$ concave in a and convex in $b$. Then there exists a point $\left(a_{0}, b_{0}\right) \in A \times B$ such that

$$
\begin{aligned}
\sup _{a \in A} \inf _{b \in B} K(a, b) & =\inf _{b \in B} K\left(a_{0}, b\right)=K\left(a_{0}, b_{0}\right) \\
& =\sup _{a \in A} K\left(a, b_{0}\right)=\inf _{b \in B} \sup _{a \in A} K(a, b) .
\end{aligned}
$$

Without loss of generality we may assume throughout that $\mu_{1}, \mu_{2}, \ldots, \mu_{n}$ are absolutely continuous with respect to the same measure $v$ (e.g. $v=\sum_{i=1}^{n} \mu_{i}$ ). For all $i \in I$, let $f_{i}=d \mu_{i} / d v$. With every $p \in \bar{S}$, where $\bar{S}$ stands for the closure of $S$ in $R^{n}$, we will associate $\mathscr{B}$-measurable subsets $B_{1}(p), B_{2}(p), \ldots, B_{n}(p)$ and $C_{1}(p)$, $C_{2}(p), \ldots, C_{n}(p)$ of $\mathscr{X}$ defined by

$$
\begin{aligned}
& B_{i}(p)=\bigcap_{\substack{j=1 \\
j \neq i}}^{n}\left\{x \in \mathscr{X}: p_{i} \alpha_{i}^{-1} f_{i}(x)>p_{j} \alpha_{j}^{-1} f_{j}(x)\right\}, \\
& C_{i}(p)=\bigcap_{j=1}^{n}\left\{x \in \mathscr{X}: p_{i} \alpha_{i}^{-1} f_{i}(x) \geq p_{j} \alpha_{j}^{-1} f_{j}(x)\right\} \quad \text { for } i \in I .
\end{aligned}
$$

Now we may state the main result of the paper.

THEOREM 3. For all $\alpha \in S$ there exist a point $p^{\alpha}=\left(p_{1}^{\alpha}, \ldots, p_{n}^{\alpha}\right) \in \bar{S}$ and a corresponding partition $P^{\alpha}=\left\{A_{i}^{\alpha}\right\}_{i=1}^{n} \in \mathscr{P}$ which satisfies

(i) $B_{i}\left(P^{\alpha}\right) \subset A_{i}^{\alpha} \subset C_{i}\left(p^{\alpha}\right)$, 
(ii) $\mu_{1}\left(A_{1}^{\alpha}\right) / \alpha_{1}=\mu_{2}\left(A_{2}^{\alpha}\right) / \alpha_{2}=\cdots=\mu_{n}\left(A_{n}^{\alpha}\right) / \alpha_{n}$ and is $\alpha$-optimal. Moreover, any partition satisfying (i) and (ii) is $\alpha$-optimal.

PROOF. It is obvious that

$$
\sup _{a \in \bar{\mu}(\mathscr{P})} \min _{i \in I} \alpha_{i}^{-1} a_{i}=\sup _{a \in \bar{\mu}(\mathscr{P})} \min _{p \in \bar{S}} \sum_{i=1}^{n} p_{i} \alpha_{i}^{-1} a_{i},
$$

where $a_{i}$ is the $i$ th coordinate of $a \in \bar{\mu}(\mathscr{P})$. Moreover, it follows from Theorem 2 that there exists a point $\left(p^{\alpha}, a^{\alpha}\right) \in \bar{S} \times \bar{\mu}(\mathscr{P})$ for which

$$
\begin{aligned}
\sup _{a \in \bar{\mu}(\mathscr{P})} \min _{p \in \bar{S}} \sum_{i=1}^{n} p_{i} \alpha_{i}^{-1} a_{i} & =\min _{p \in \bar{S}} \sum_{i=1}^{n} p_{i} \alpha_{i}^{-1} a_{i}^{\alpha}=\sum_{i=1}^{n} p_{i}^{\alpha} \alpha_{i}^{-1} a_{i}^{\alpha} \\
& =\sup _{a \in \bar{\mu}(\mathscr{P})} \sum_{i=1}^{n} p_{i}^{\alpha} \alpha_{i}^{-1} a_{i}=\min _{p \in \bar{S}} \sup _{a \in \bar{\mu}(\mathscr{P})} \sum_{i=1}^{n} p_{i} \alpha_{i}^{-1} a_{i} .
\end{aligned}
$$

It is clear that any partition $P^{\alpha}=\left\{A_{i}^{\alpha}\right\}_{i=1}^{n} \in \mathscr{P}$ with $\mu_{i}\left(A_{i}^{\alpha}\right)=a_{i}$ for $i \in I$ is $\alpha$-optimal. Now, since by (1)

$$
\sum_{i=1}^{n} p_{i}^{\alpha} \alpha_{i}^{-1} \mu_{i}\left(A_{i}^{\alpha}\right)=\sup _{P \in \mathscr{P}} \sum_{i=1}^{n} p_{i}^{\alpha} \alpha_{i}^{-1} \mu_{i}\left(A_{i}\right)
$$

and since

$$
\min _{p \in \bar{S}} \sup _{P \in \mathscr{P}} \sum_{i=1}^{n} p_{i} \alpha_{i}^{-1} \mu_{i}\left(A_{i}\right)=\min _{p \in \bar{S}} \sum_{i=1}^{n} p_{i} \alpha_{i}^{-1} \mu_{i}\left(A_{i}^{\alpha}\right)
$$

it follows that (i) and (ii) must be fulfilled, respectively. This completes the proof of the first part of the Theorem. The proof of the second part is straightforward.

\section{REFERENCES}

1. J. P. Aubin, Mathematical methods of game and economic theory, North-Holland, 1979, pp. 204-225.

2. L. Dubins and E. Spanier, How to cut a cake fairly, Amer. Math. Monthly 68 (1961), 1-17.

3. A. Dvoretzky, A. Wald and J. Wolfowitz, Relations among certain ranges of vector measures, Pacific J. Math. 1 (1951), 59-74.

4. J. Elton, T. Hill and R. Kertz, Optimal partitioning inequalities for nonatomic probability measures, Trans. Amer. Math. Soc. 296 (1986), 703-725.

5. B. Knaster, Sur le probleme du partage pragmatique de H. Steinhaus, Ann. Soc. Polon. Math. 19 (1946), 228-230.

6. J. Legut, Inequalities for $\alpha$-optimal partitioning of a measurable space, Proc. Amer. Math. Soc. (to appear).

7. K. Urbanik, Quelques theoremes sur les measures, Fund. Math. 41 (1954), 150-162.

8. D. Weller, Fair division of a measurable space, J. Math. Econ. 14 (1985), 5-17.

9. M. Wilczyński, Optimal partitioning inequalities for nonatomic finite measures, unpublished result.

Institute of Mathematics, Technical University of Wroceaw, Wybrzeźe WYSPIAŃSKIEGO 27, 50-370 WROClaW, POLAND 